\title{
Protection of Gynostemma pentaphyllum Extract on Hemolysis-Induced by Plasmodium berghei Infection in ICR Mice
}

\author{
Preeyanuch Borkaew ${ }^{1}$, Chokdee Klubsri ${ }^{2}$, Voravuth Somsak*3 \\ Department of Clinical Chemistry, Faculty of Medical Technology, \\ Western University, Kanchanaburi 71170, Thailand
}

Abstract: Hemolysis is one manifestation of Plasmodium parasite infection and well-known causes of death in severe malaria. The present study was aimed to investigate protective effect of Gynostemma pentaphyllum extract on hemolysis-induced by Plasmodium berghei infection in mice. Aqueous crude extract of $G$. pentaphyllum leaves was prepared using hot water method. Standard 4day test was used to evaluate the efficacy of this extract on hemolysis during malaria infection. Groups of ICR mice (5 mice of each) were inoculated with $1 \times 10^{7}$ parasitized erythrocytes of P. berghei ANKA (PbANKA) by intraperitoneal injection, and subsequently given the extract $(100,1,000$, and $3,000 \mathrm{mg} / \mathrm{kg}$ ) orally by gavage twice a day for 4-consecutive days. The results showed that aqueous crude extract of $G$. pentaphyllum leaves exhibited significant $(p<0.05)$ dose-dependent protection of hemolysis-induced by PbANKA. The maximum activity was observed at a dose of 3,000 mg/kg extract. Additionally, no any toxicities were found in normal mice treated with this extract. It can be concluded that aqueous crude extract of $G$. pentaphyllum leaves exerted protective effect on hemolysis-induced by malaria infection.

Keywords: Gynostemma pentaphyllum, hemolysis, Plasmodium berghei, mice

\section{Introduction}

Malaria-associated hemolysis, one of the major life-threatening well-known causes of death in severe malaria, occurs between $1-4 \%$ of hospitalized adult with a mortality that can reach up to $45 \%$ [1]. The pathogenesis of malariaassociated hemolysis is multifactorial, but several hypotheses suggest involvement of cytoadherence of parasitized erythrocytes, inflammatory response as well as oxidative stress [2]. Hence, finding the medicinal plant extracts to protect hemolysis during malaria infection is urgently needed.

Gynostemma pentaphyllum (Cucurbitaceae), known as Jiaogulan, is a perennial vine endemic in China, Japan, India, Korea, and Thailand [3]. Recently, this plant has attracted great attention owing to its antioxidant, anti-inflammation, anticancer, immunomodulative, anti-bacterial, homeostasis of blood glucose and lipid, and protection of organ damage and hemolysis induced by oxidative stress [4-8]. However, protection of hemolysis during malaria infection of $G$. pentaphyllum extract has not yet been reported.

According to this, the main focus of this study was to evaluate protective effect of $G$. pentaphyllum extract on hemolysis-induced by $P$. berghei infection in mice.

\section{Methodologies}

\subsection{Preparation of aqueous crude extract}

Dried powdered of $G$. pentaphyllum leaves was purchased from the Royal Project Foundation shop at Suphanburi province, Thailand. The extraction was performed by mixing the dried powdered plant material in distilled water (DW) at the proportion of 1:10. Microwave at $360 \mathrm{~W}$ for $5 \mathrm{~min}$ was then used to heat, and incubated at room temperature for $3 \mathrm{~h}$. Filtration was subsequently performed through Whatman no. 1 filter paper, and filtrate 
was collected. Freeze-drying was carried out to obtained aqueous dried powdered crude extract of G. pentaphyllum (GPE), and stored at $-20^{\circ} \mathrm{C}$. Before using, the GPE was dissolved completely in DW [9].

\subsection{Experimental mice}

Female ICR mice, 4-6 weeks old, weighting 25-30 $\mathrm{g}$, obtained from the National Laboratory Animal Center, Mahidol University, Bangkok, Thailand, were used. They were kept at the room with temperature control between $25-28^{\circ} \mathrm{C}$, and fed with standard pellet diet and clean water ad libitum. Experiments involving the animals were ratified by the Animal Ethical Committee, Western University, Thailand.

\subsection{Rodent malaria parasite}

Plasmodium berghei ANKA strain (PbANKA) was used in this study. Parasite was maintained by mechanical intraperitoneal passage in ICR mice. Percentage of packed cell volume (\% PCV) was also measured.

\subsection{Efficacy test of G. pentaphyllum in mice}

Standard 4-day test was carried out as previously described [10]. Groups of ICR mice (5 mice of each) were inoculated with $1 \times 10^{7}$ parasitized erythrocytes of PbANKA by intraperitoneal injection. They were subsequently administered with $100,1,000$, and 3,000 mg/kg of GPE orally by gavage twice a day for 4-consecutive days (Day03). At day 4, \% PCV was measured. Three controls including naïve ICR mice treated with or without $3,000 \mathrm{mg} / \mathrm{kg}$ of GPE, and PbANKA infected mice were used as healthy, toxicity, and untreated controls.

\subsection{Determination of mean survival time}

Mortality was monitored daily and number of the days from the time of infection up to death was recorded for each mouse in the treatment and control groups throughout the follow-up period and the mean survival time (MST) was then calculated for each group by using the following formula [11].

\section{MST $=\underline{\text { Sum of survival time of all mice }}$}

Total number of mice

\subsection{Statistics}

All results were expressed as mean \pm standard error of mean (SEM). Significant level considered at $95 \%$ confidence, $p<0.05$ was analyzed with oneway ANOVA with Tukey post-hoc test.

\section{Results}

\subsection{Malaria-associated hemolysis}

Parasitemia was first detectable on day 1 postinfection with a parasitemia less than $1 \%$. However, parasitemia reached $70 \%$ on day 12 post-infection with infected mice died. Moreover, we observed that increase of hemolysis was found during malaria infection in mice as indicated by markedly decreasing of \% PCV (Figure 1).

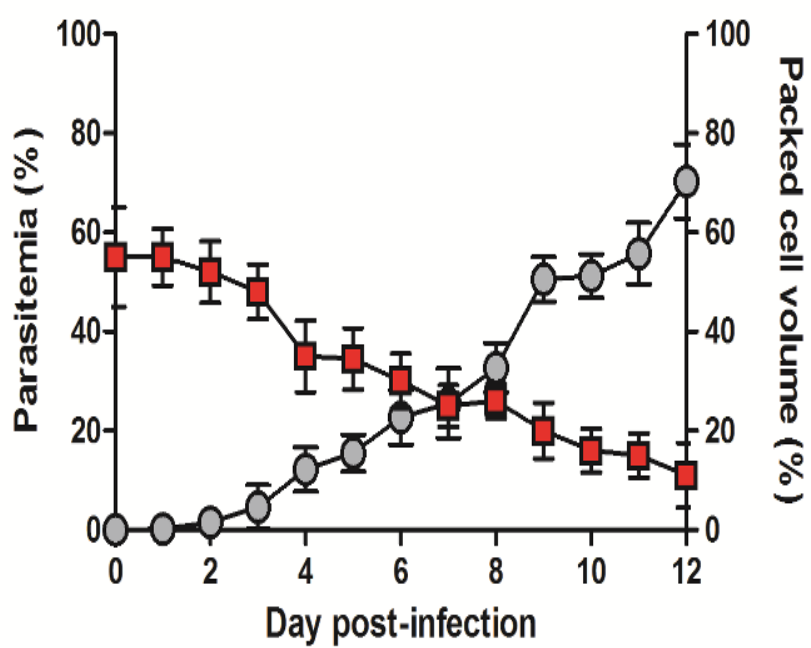

Figure 1: Malaria-associated hemolysis

3.2 Protective effect of GPE on hemolysisinduced by PbANKA infection

During PbANKA infection in mice, \% PCV was significantly $(p<0.001)$ decreased, compared to 
normal mice $(\mathrm{N})$. Interestingly, aqueous crude extract of $G$. pentaphyllum leaves exerted a dosedependent protection of hemolysis-induced by malaria infection in mice. The extract caused a significant $(p<0.01$ and $p<0.001$ at doses of 1,000 and 3,000, respectively) protection, compared to untreated control (UN). The highest activity was found at the dose of $3,000 \mathrm{mg} / \mathrm{kg}$. Moreover, this extract has no effect on hemolysis in normal mice (N+GPE) (Figure 2).

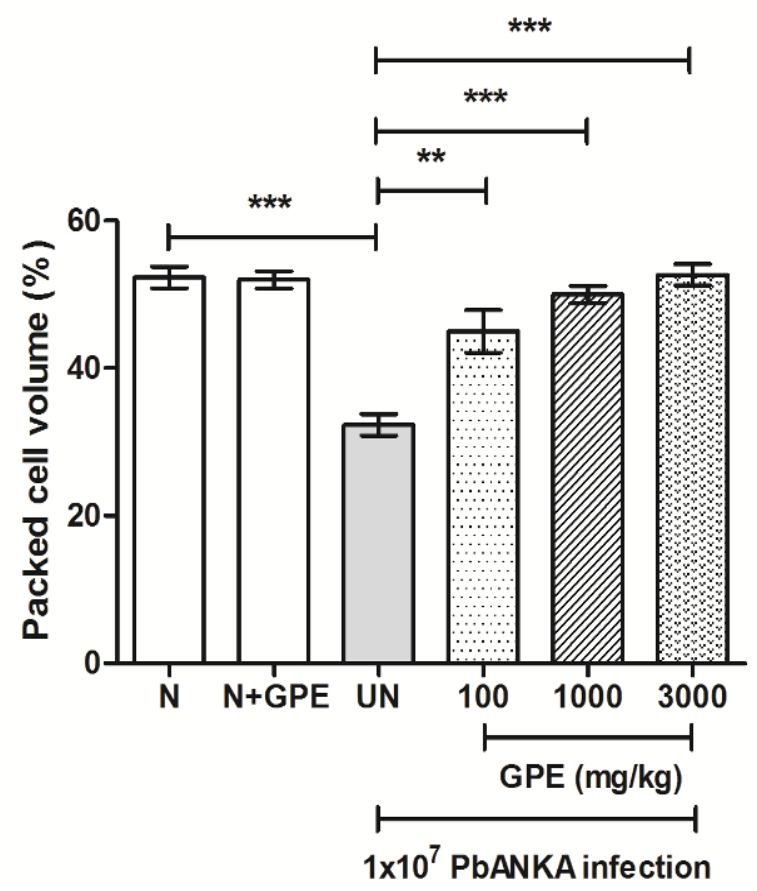

Figure 2: Protection of GPE on hemolysisinduced by PbANKA infection

\subsection{Effect of GPE on survival time of mice}

It was found that $100,1,000$, and $3,000 \mathrm{mg} / \mathrm{kg}$ of GPE treated mice lived longer $(25,32$, and 35 days, respectively) than the corresponding untreated control (12 days). There was no significant differences in survival times among extract treated groups.

\section{Discussions}

Protective effect of the aqueous crude extract of $G$. pentaphyllum leaves on hemolysis-induced by $P$. berghei infection in mice was reported. During malaria infection, hemolysis was developed as indicated by markedly decrease of \% PCV. Several hypotheses suggested the involvement of cytoadherence of parasitized erythrocytes, proinflammatory response as well as oxidative stress [12]. During erythrocytic phase, the consumption of hemoglobin by parasites gives rise of considerable amounts of free heme, a molecule that have the ability to induce oxidative stress and hemolysis [13]. Moreover, malaria-associated hemolysis is proposed to be a consequence of parasite adhesion as well as exacerbated immune response against products of oxidative stress released during infection [14]. In addition, inhibition of erythropoietin function during malaria infection in blood stage has also been discussed [15].

The present study showed that protection of hemolysis-induced by malaria infection in mice was observed in infected mice treated with $G$. pentaphyllum extract. This extract has been reported to have potent antioxidant, antiinflammation, and protection of organ damage and hemolysis induced by oxidative stress [4]. Therefore, our data might add new insights to previous findings demonstrating hemolysisinduced by malaria. Moreover, destruction of erythrocytes in both infected and normal ones might be prevented by aqueous crude extract of $G$. pentaphyllum leaves via its antioxidant activity and erythrocyte membrane protection. Therefore, the antioxidant and free radical scavenging activities of this extract might inhibit oxidative stress and protect erythrocytes during malaria infection. It has also been described that erythropoietin was activated and increased in the extract treatment. So, increasing of erythropoietin and erythrocyte production during malaria infection by this extract might play another role to prevent hemolysis [15].

Mean survival time is another parameter evaluates the activity of plant extracts. Accordingly, a plant extract that can prolong the survival time of infected mice compared to untreated control are considered as active against malaria. In this study, 
the infected mice treated with $G$. pentaphyllum extract had significantly lived longer than negative control. This might be due to the anti-hemolysis activity of the extract.

\section{Conclusion}

This study has shown that aqueous crude extract of G. pentaphyllum leaves exhibited the protective effect on hemolysis-induced by malaria infection. Further studies are recommended on antihemolytic activity as well as safety profiles of this plant.

\section{Acknowledgements}

This study was financially supported by the Western University. The authors would like to acknowledge all students at the Department of Clinical Chemistry, Faculty of Medical Technology, Western University. We also special thank Dr. Chairat Uthaipibull from BIOTEC, and Assoc. Prof. Dr. Somdet Srichairatanakool from Chiang Mai University for their excellent discussion.

\section{References}

[1] Khobjai W, Jaihan U, Watcharasamphankul W, Somsak V. Protective effect of Thunbergia laurifolia extract on hemolysis during Plasmodium berghei infection. Parasitology research. 2014;113:1843-6.

[2] Elias RM, Correa-Costa M, Barreto CR, Silva RC, Hayashida CY, Castoldi A, et al. Oxidative stress and modification of renal vascular permeability are associated with acute kidney injury during Plasmodium berghei ANKA infection. PloS one. 2012;7:e44004.

[3] Cui J, Eneroth P, Bruhn JG. Gynostemma pentaphyllum: identification of major sapogenins and differentiation from Panax species. European journal of pharmaceutical sciences : official journal of the European Federation for Pharmaceutical Sciences. 1999;8:187-91.
[4] Lin CC, Huang PC, Lin JM. Antioxidant and hepatoprotective effects of Anoectochilus formosanus and Gynostemma pentaphyllum. The American journal of Chinese medicine. 2000;28:87-96.

[5] Xie Z, Huang H, Zhao Y, Shi H, Wang S, Wang TT, et al. Chemical composition and antiproliferative and anti-inflammatory effects of the leaf and whole-plant samples of diploid and tetraploid Gynostemma pentaphyllum (Thunb.) Makino. Food chemistry. 2012;132:125-33.

[6] Yan H, Wang X, Niu J, Wang Y, Wang P, Liu Q. Anti-cancer effect and the underlying mechanisms of gypenosides on human colorectal cancer SW-480 cells. PloS one. 2014;9:e95609.

[7] Srichana D, Taengtip R, Kondo S. Antimicrobial activity of Gynostemma pentaphyllum extracts against fungi producing aflatoxin and fumonisin and bacteria causing diarrheal disease. The Southeast Asian journal of tropical medicine and public health 2011;42:704-10.

[8] Sun H, Zheng Q. Haemolytic activities and adjuvant effect of Gynostemma pentaphyllum saponins on the immune responses to ovalbumin in mice. Phytotherapy research : PTR. 2005;19:895-900.

[9] Song SL, Tang JB, Ji AG, Liang H, Zhu P, Wang WL. The extraction, purification and assaying of Gynostemma pentaphyllum polysaccharides. Journal of Chinese medicinal materials. 2006;29:595-8.

[10] Peters W. The chemotherapy of rodent malaria, XXII. The value of drug-resistant strains of Plasmodium berghei in screening for blood schizontocidal activity. Annals of tropical medicine and parasitology. 1975;69:155-71.

[11] Kefe A, Giday M, Mamo H, Erko B. Antimalarial properties of crude extracts of seeds of Brucea antidysenterica and leaves of Ocimum lamiifolium. BMC complementary and alternative medicine. 2016;16:118.

[12] Nutham N, Sakulmettatham S, Klongthalay S, Chutoam P, Somsak V. Protective Effects of 
Tinospora crispa Stem Extract on Renal

Damage and Hemolysis during Plasmodium berghei Infection in Mice. Journal of pathogens. 2015;2015:738608.

[13] Wood PA, Eaton JW. Hemoglobin catabolism and host-parasite heme balance in chloroquine-sensitive and chloroquine-resistant Plasmodium berghei infections. The American journal of tropical medicine and hygiene. 1993;48:465-72.

[14] Loose LD, di Luzio NR. A temporal relationship between reticuloendothelial system phagocytic alterations and antibody responses in mice infected with Plasmodium berghei (NYU-2 strain). The American journal of tropical medicine and hygiene. 1976;25:221-8.

[15] Core A, Hempel C, Kurtzhals JA, Penkowa M. Plasmodium berghei ANKA: erythropoietin activates neural stem cells in an experimental cerebral malaria model. Experimental parasitology. 2011;127:500-5. 